\title{
Pacific
}

Journal of

Mathematics

CONGRUENCE PROPERTIES OF FUNCTIONS RELATED TO THE PARTITION FUNCTION

ANTHONY D. FORBES 


\section{CONGRUENCE PROPERTIES OF FUNCTIONS RELATED TO THE PARTITION FUNCTION}

\section{ANTHONY D. Forbes}

In this paper we describe a straightforward and almost entirely elementary method for establishing congruence properties of certain functions that are related to the partition function.

For integer $k$ define $p_{k}(n)$ by

$$
\prod_{m=1}^{\infty}\left(1-x^{m}\right)^{k}=\sum_{n=0}^{\infty} p_{k}(n) x^{n} .
$$

In particular, $p_{-1}(n)$ is $p(n)$, the partition function and $p_{24}(n-1)$ is Ramanujan's $\tau$-function.

We are interested in congruences of the form

$$
p_{k}(n p+b) \equiv 0 \quad(\bmod p) \text { for all } n \geq 1
$$

for prime $p$, as typified by the partition congruences

$$
\begin{aligned}
& p(5 n+4) \equiv 0 \quad(\bmod 5), \\
& p(7 n+5) \equiv 0 \quad(\bmod 7)
\end{aligned}
$$

and

$$
p(11 n+6) \equiv 0 \quad(\bmod 11)
$$

discovered by Ramanujan and proved in [13] and [14]. Ramanujan also conjectured that if $24 b \equiv 1(\bmod q)$ and $q=5^{\alpha} 7^{\beta} 11^{\gamma}$ then $p(q n+b) \equiv 0(\bmod q)$. He was able to supply proofs for $q=25,49$ in [13] and $q=121$ in an unpublished manuscript [15]. Ramanujan's conjecture was incorrect as stated for powers of 7 and Watson [16] proved a modified version; if $24 b \equiv 1\left(\bmod 5^{\alpha} 7^{2 \beta}\right)$ then $p\left(5^{\alpha} 7^{2 \beta} n+b\right) \equiv 0\left(\bmod 5^{\alpha} 7^{\beta+1}\right)$. Watson's proofs have been simplified by Hirschhorn and Hunt [6] and Garvan [4]. Lehner [9] dealt with $q=1331$ and the proof of the conjecture was completed by Atkin [1].

Congruences modulo powers of 13 have been considered by Atkin and O'Brien [2]. A general treatment of $p_{k}(n)$ modulo powers of $2,3,5,7$ and 13 is given in Atkin [3], modulo powers of 11 in 
Gordon [5] and modulo powers of 17 in a forthcoming paper by Hughes [7].

In everything that follows, $p$ is a prime number $\geq 5$. The variable $x$ always satisfies $|x|<1$ to ensure absolute convergence and we write $f(x) \equiv g(x)(\bmod p)$ to mean that $f(x)-g(x)$ is a power series in $x$ with integer coefficients that are all divisible by $p$.

Euler's pentagonal number theorem,

$$
\prod_{m=1}^{\infty}\left(1-x^{m}\right)=\sum_{n=-\infty}^{\infty}(-1)^{n} x^{\left(3 n^{2}+n\right) / 2},
$$

and Jacobi's identity,

$$
\prod_{m=1}^{\infty}\left(1-x^{m}\right)^{3}=\sum_{n=0}^{\infty}(-1)^{n}(2 n+1) x^{\left(n^{2}+n\right) / 2}
$$

completely determine $p_{1}(n)$ and $p_{3}(n)$. Also it suffices to consider $k$ modulo $p$ because, as is easily shown, if $p_{k}(n)$ satisfies a congruence of the form (1) for some prime $p$ then the same is true for $p_{k \pm p}(n)$.

With certain values of $k$, other than 0,1 and 3 , it is possible to establish congruences by well-known methods which are entirely elementary. For instance, Ramanujan's original proofs of (2) and (3) in [13] are easily extended to show that (1) holds when

$$
\begin{array}{lllll}
k=4, & p \equiv 5 & (\bmod 6), & 6 b+1 \equiv 0 & (\bmod p) \\
k=6, & p \equiv 3 & (\bmod 4), & 4 b+1 \equiv 0 & (\bmod p) .
\end{array}
$$

For an alternative proof of (2), the congruence

$$
p_{9}(5 m+4) \equiv 0 \quad(\bmod 5),
$$

follows from

$$
p_{9}(n)=\sum_{r=0}^{n} \sum_{s=0}^{n-r} p_{3}(r) p_{3}(s) p_{3}(n-r-s) .
$$

By $(5)$, if $n \equiv 4(\bmod 5)$ and the $r, s$ term of the double sum is non-zero then

$$
p_{3}(r)^{2}+p_{3}(s)^{2}+p_{3}(n-r-s)^{2}=8 n+3 \equiv 0 \quad(\bmod 5),
$$

which cannot be true unless at least one of the terms on the left-hand side is divisible by 5 . But then $p_{9}(n)$ will also be a multiple of 5 .

In Table 1 we give an exhaustive list of congruences of the form (1) for $p \leq 199$ and $2 \leq k \leq p-1, k \neq 3,4,6$. 
A theorem of Newman [10] established using modular function theory states that if $k=4,6,8,10,14,26, p$ is a prime $>3$ such that $k(p+1) \equiv 0(\bmod 24)$ and $b=k\left(p^{2}-1\right) / 24$ then $p_{k}(n) \equiv 0(\bmod p)$ for $n \equiv b(\bmod p)$. This theorem disposes of all the $k=8$ cases in Table 1 as well as $k=10,14$ and 26 when $p \equiv 11(\bmod 12)$. Another of Newman's results [11] is that for even $k, 4 \leq k \leq 24$ and prime $p>3$ such that $b=k(p-1) / 24$ is an integer,

$$
p_{k}(n p+b) \equiv p_{k}(n) p_{k}(b) \quad(\bmod p) .
$$

Thus $k=19, p=12$ and $k=22, p=61$ in Table 1 reduce to single congruences. Newman's method is described in Chapter 7 of Knopp [8].

In [14], Ramanujan gives proofs of (4) by two different methods one of which we extend in order to deal with any congruence of the form $(1)$ for which $24 b+k \equiv 0(\bmod p)$. In particular we can prove all the entries in Table 1 (see next page).

We illustrate the method with $k=10, p=19, b=17$ and for convenience we use the same notation as Ramanujan. Let

$$
\begin{aligned}
\phi_{r, s}(x) & =\sum_{m=1}^{\infty} \sum_{n=1}^{\infty} m^{r} n^{s} x^{m n}, \\
P & =1-24 \phi_{0,1}(x), \\
Q & =1+240 \phi_{0,3}(x)
\end{aligned}
$$

and

$$
R=1-504 \phi_{0,5}(x) .
$$

It is well known from the theory of the Dedekind eta-function that

$$
12^{3} x \prod_{m=1}^{\infty}\left(1-x^{m}\right)^{24}=Q^{3}-R^{2} .
$$

In fact, $P, Q$ and $R$ are the normalised Eisenstein series $E_{2}, E_{4}$ and $E_{6}$. They are related to the discriminant $\Delta$ and the invariants $g_{2}(\tau)$ and $g_{3}(\tau)$ by

$$
P=\frac{1}{2 \pi i} \frac{\Delta^{\prime}(\tau)}{\Delta(\tau)}, \quad Q=\frac{3}{4} \frac{g_{2}(\tau)}{\pi^{4}} \quad \text { and } \quad R=\frac{27}{8} \frac{g_{3}(\tau)}{\pi^{6}},
$$

where $x=e^{2 \pi i \tau}$ for $\tau$ in the upper half plane. 
TABLE 1

$$
p_{k}(n p+b) \equiv 0(\bmod p)
$$

\begin{tabular}{|c|c|c|c|c|c|c|c|}
\hline \multirow[b]{2}{*}{$p$} & \multicolumn{7}{|c|}{$k$} \\
\hline & 8 & 10 & 12 & 14 & 18 & 22 & 26 \\
\hline 11 & 7 & 6 & - & - & - & - & - \\
\hline 17 & 11 & & & 15 & - & - & - \\
\hline 19 & & 17 & 9 & & & - & - \\
\hline 23 & 15 & 13 & & 9 & 5 & & - \\
\hline 29 & 19 & & & 26 & & & \\
\hline 31 & & 28 & & & & & \\
\hline 41 & 27 & & & 37 & & & \\
\hline 43 & & 39 & & & & & \\
\hline 47 & 31 & 27 & & 19 & & & 42 \\
\hline 53 & 35 & & & 48 & & & \\
\hline 59 & 39 & 34 & & 24 & & & 53 \\
\hline 61 & & & & & & 55 & \\
\hline 67 & & 61 & & & & & \\
\hline 71 & 47 & 41 & & 29 & & & 64 \\
\hline 79 & & 72 & & & & & \\
\hline 83 & 55 & 48 & & 34 & & & 75 \\
\hline 89 & 59 & & & 81 & & & \\
\hline 101 & 67 & & & 92 & & & \\
\hline 103 & & 94 & & & & & \\
\hline 107 & 71 & 62 & & 44 & & & 97 \\
\hline 113 & 75 & & & 103 & & & \\
\hline 127 & & 116 & & & & & \\
\hline 131 & 87 & 76 & & 54 & & & 119 \\
\hline 137 & 91 & & & 125 & & & \\
\hline 139 & & 127 & & & & & \\
\hline 149 & 99 & & & 136 & & & \\
\hline 151 & & 138 & & & & & \\
\hline 163 & & 149 & & & & & \\
\hline 167 & 111 & 97 & & 69 & & & 152 \\
\hline 173 & 115 & & & 158 & & & \\
\hline 179 & 119 & 104 & & 74 & & & 163 \\
\hline 191 & 127 & 111 & & 79 & & & 174 \\
\hline 197 & 131 & & & 180 & & & \\
\hline 199 & & 182 & & & & & \\
\hline
\end{tabular}

In [12], Ramanujan establishes in a direct and elementary manner a number of identities involving $P, Q$ and $R$, including

and

$$
\begin{gathered}
Q R=1-264 \phi_{0,9}(x), \\
441 Q^{3}+250 R^{2}=691+65520 \phi_{0,11}(x), \\
P^{2}-Q=12 \theta P \\
P Q-R=3 \theta Q
\end{gathered}
$$

where $\theta$ is the differential operator $x d / d x$. 
Now to prove that $P_{10}(19 n+17) \equiv 0(\bmod 19)$ for all $n \geq 0$, it suffices to show that the same is true for $p_{48}(19 n+17)$. By (6) this is equivalent to showing that in

$$
\left(Q^{3}-R^{2}\right)^{2}=\sum_{n=1}^{\infty} c(n) x^{n}
$$

the coefficients $c(19), c(38), \ldots$ are multiples of 19 and one way of doing this is to find a power series $f(x)$ with integer coefficients satisfying

$$
\left(Q^{3}-R^{2}\right)^{2} \equiv 12 \theta f(x) \quad(\bmod 19) .
$$

We succeed because of the identity

$$
\begin{array}{r}
12 \theta\left(9 P^{3} Q^{4}+16 P^{3} Q R^{2}+13 P^{2} Q^{3} R+7 P^{2} R^{3}\right. \\
\left.+5 P Q^{5}+13 P Q^{2} R^{2}+18 Q^{4} R+14 Q R^{3}\right) \\
=\left(Q^{3}-R^{2}\right)^{2}+19\left(9 P^{4} Q^{4}+16 P^{4} Q R^{2}-4 P^{3} Q^{3} R+4 P^{3} R^{3}\right. \\
-3 P^{2} Q^{2} R^{2}+6 P Q^{4} R+10 P Q R^{3} \\
\left.-6 Q^{6}-29 Q^{3} R^{2}-3 R^{4}\right)
\end{array}
$$

which is easily verified using (9), (10) and (11).

To obtain an identity like (12) we consider the matrix $A_{\lambda, \mu, \nu}^{\alpha, \beta, \gamma}$ defined by equating coefficients of $P^{\lambda} Q^{\mu} R^{\nu}$ in

$$
\sum_{\substack{\lambda, \mu, \nu \geq 0 \\ \lambda+2 \mu+3 \nu=6 s}} P^{\lambda} Q^{\mu} R^{\nu} A_{\lambda, \mu, \nu}^{\alpha, \beta, \nu}=12 \theta P^{\lambda} Q^{\mu} R^{\nu}
$$

as $\alpha, \beta$ and $\gamma$ run through the non-negative integers satisfying $\alpha+$ $2 \beta+3 \gamma=6 s-1$. Here $s$ satisfies

$$
24 s \equiv k \quad(\bmod p) .
$$

Next we solve the linear congruences

$$
\sum_{\substack{\alpha, \beta, \gamma \geq 0 \\ \alpha+2 \beta+3 \gamma=6 s-1}} A_{\lambda, \mu, \nu}^{\alpha, \beta, \gamma} a_{\alpha, \beta, \gamma} \equiv t_{\lambda, \mu, \nu} \quad(\bmod p)
$$

for $a_{\alpha, \beta, \gamma}$, where

$$
\begin{aligned}
& t_{0, \mu, \nu}=(-1)^{\nu / 2}\left(\begin{array}{c}
s \\
\nu / 2
\end{array}\right), \\
& t_{\lambda, \mu, \nu}=0 \quad \text { for } \lambda \geq 1
\end{aligned}
$$


TABLE 2

$12 \theta P^{\alpha} Q^{\beta} R^{\gamma}$ for $\alpha+2 \beta+3 \gamma=11$

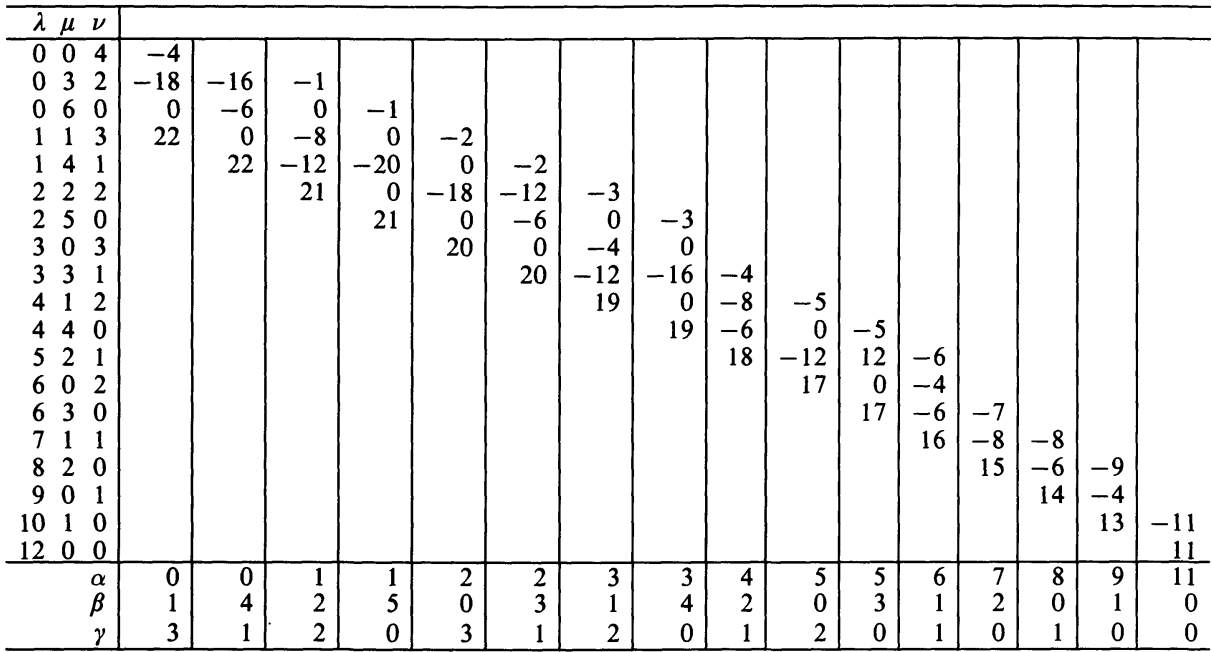

and, as before, $\lambda+2 \mu+3 \nu=6 s$. Then $a_{\alpha, \beta, \gamma}$ are the required coefficients, for

$$
\begin{aligned}
& 12 \theta \sum_{\substack{\alpha, \beta, \gamma \geq 0 \\
\alpha+2 \beta+3 \gamma=6 s-1}} a_{\alpha, \beta, \gamma} P^{\alpha} Q^{\beta} R^{\gamma} \\
& \equiv \sum_{\substack{\alpha, \beta, \gamma \geq 0 \\
\alpha+2 \beta+3 \gamma=6 s-1}} \sum_{\substack{\lambda, \mu, \nu \geq 0 \\
\lambda+2 \mu+3 \nu=6 s}} P^{\lambda} Q^{\mu} R^{\nu} A_{\lambda, \mu, \nu}^{\alpha, \beta, \gamma} a_{\alpha, \beta, \gamma} \\
& \equiv \sum_{\substack{\lambda, \mu, \nu \geq 0 \\
\lambda+2 \mu+3 \nu=6 s}} t_{\lambda, \mu, \nu} P^{\lambda} Q^{\mu} R^{\nu} \equiv\left(Q^{3}-R^{2}\right)^{s} \quad(\bmod p) .
\end{aligned}
$$

The case $s=2$ is illustrated in Table 2 .

What is interesting is perhaps not the actual method, for it merely involves routine computations, but rather the existence of the identity itself. It seems that there is no simpler expression of the form $12 \theta f(x)$ that will serve our purpose.

In the other case for $p=19$, namely $k=12$, the corresponding expression is somewhat longer. The exponent of $Q^{3}-R^{2}$ is 10 and we are dealing with $P^{\alpha} Q^{\beta} R^{\gamma}$ where $\alpha+2 \beta+3 \gamma=59$. The result of solving the congruences is 


$$
\begin{aligned}
& 12 \theta\left(4 P^{4} Q^{26} R+16 P^{4} Q^{23} R^{3}+12 P^{4} Q^{20} R^{5}+17 P^{4} Q^{17} R^{7}\right. \\
& +10 P^{4} Q^{14} R^{9}+8 P^{4} Q^{11} R^{11}+16 P^{4} Q^{8} R^{13}+6 P^{4} Q^{5} R^{15} \\
& +13 P^{4} Q^{2} R^{17}+5 P^{3} Q^{28}+P^{3} Q^{25} R^{2}+8 P^{3} Q^{22} R^{4} \\
& +2 P^{3} Q^{19} R^{6}+5 P^{3} Q^{16} R^{8}+5 P^{3} Q^{13} R^{10}+4 P^{3} Q^{10} R^{12} \\
& +7 P^{3} Q^{7} R^{14}+2 P^{3} Q^{4} R^{16}+9 P^{3} Q R^{18}+9 P^{2} Q^{27} R \\
& +7 P^{2} Q^{24} R^{3}+13 P^{2} Q^{21} R^{5}+2 P^{2} Q^{18} R^{7}+7 P^{2} Q^{15} R^{9} \\
& +5 P^{2} Q^{12} R^{11}+7 P^{2} Q^{9} R^{13}+16 P^{2} Q^{6} R^{15}+15 P^{2} Q^{3} R^{17} \\
& +18 P^{2} R^{19}+4 P Q^{29}+6 P Q^{26} R^{2}+P Q^{23} R^{4} \\
& +14 P Q^{20} R^{6}+8 P Q^{17} R^{8}+8 P Q^{14} R^{10}+P Q^{8} R^{14} \\
& +13 P Q^{5} R^{16}+12 P Q^{2} R^{18}+15 Q^{28} R+4 Q^{25} R^{3} \\
& +13 Q^{22} R^{5}+16 Q^{19} R^{7}+3 Q^{16} R^{9}+10 Q^{13} R^{11} \\
& \left.+15 Q^{10} R^{13}+5 Q^{7} R^{15}+7 Q^{4} R^{17}+14 Q R^{19}\right) \\
& \equiv\left(Q^{3}-R^{2}\right)^{10} \quad(\bmod 19) \text {. }
\end{aligned}
$$

In one of his proofs of (4), Ramanujan uses (7) and (8) as well as

$$
\begin{gathered}
Q(P Q-R)=720 \phi_{1,8}(x) \\
2 P Q^{2}-P^{2} R-Q R=1728 \phi_{2,7}(x) \\
P^{3} Q-3 P^{2} R+3 P Q^{2}-Q R=3456 \phi_{3,6}(x)
\end{gathered}
$$

and

$$
15 P Q^{2}-20 P^{2} R+10 P^{3} Q-4 Q R-P^{5}=20736 \phi_{4,5}(x)
$$

in order to establish

$$
\left(Q^{3}-R^{2}\right)^{5} \equiv-5 \phi_{1,8}(x)+3 \phi_{2,7}(x)+3 \phi_{3,6}(x)-\phi_{4,5}(x) \quad(\bmod 11)
$$

in which it is clear that the coefficients of $x^{11 n}$ on the right-hand side are divisible by 11 .

Alternatively, using our method we obtain

$$
\begin{aligned}
12 \theta( & 10 P^{3} Q^{13}+P^{3} Q^{10} R^{2}+7 P^{3} Q^{7} R^{4}+7 P^{3} Q^{4} R^{6}+5 P^{3} Q R^{8} \\
& +4 P^{2} Q^{12} R+10 P^{2} Q^{9} R^{3}+8 P^{2} Q^{6} R^{5}+9 P^{2} R^{9}+5 P Q^{14} \\
& +6 P Q^{11} R^{2}+8 P Q^{8} R^{4}+2 P Q^{5} R^{6}+3 P Q^{2} R^{8} \\
& \left.+10 Q^{13} R+Q^{10} R^{3}+Q^{7} R^{5}+10 Q^{4} R^{7}+3 Q R^{9}\right) \\
\equiv & \left(Q^{3}-R^{2}\right)^{5} \quad(\bmod 11) .
\end{aligned}
$$


For the other $p=11$ case, namely $k=8, b=7$ we use

$$
\begin{aligned}
12 \theta & \left(3 P^{2} Q^{9} R+9 P^{2} Q^{6} R^{3}+8 P^{2} Q^{3} R^{5}+5 P^{2} R^{7}+7 P Q^{11}\right. \\
& \left.+6 P Q^{8} R^{2}+5 P Q^{5} R^{4}+9 P Q^{2} R^{6}+6 Q^{10} R+8 Q R^{7}\right) \\
& \equiv\left(Q^{3}-R^{2}\right)^{4} \quad(\bmod 11) .
\end{aligned}
$$

In a similar manner we can complete the proof of all the congruences in Table 1 except for $k=26, p \neq 179$ where, as can be verified by computation, it turns out that there is no formula of the form

$$
12 \theta \sum_{\alpha, \beta, \gamma} a_{\alpha, \beta, \gamma} P^{\alpha} Q^{\beta} R^{\gamma} \equiv\left(Q^{3}-R^{2}\right)^{p-b} \quad(\bmod p) .
$$

In fact we obtain

$$
\begin{aligned}
& 12 \theta \sum_{\alpha, \beta, \gamma} a_{\alpha, \beta, \gamma} P^{\alpha} Q^{\beta} R^{\gamma} \\
& \quad \equiv\left(Q^{3}-R^{2}\right)^{(p+13) / 12}+u(p) P^{11} Q^{(p-27) / 4} R\left(Q^{3}-R^{2}\right)
\end{aligned}
$$

$(\bmod p)$

for some $a_{\alpha, \beta, \gamma}$ and $u(p)(\bmod p)$. As noted above, $u(179)=0$.

Nevertheless, using the same method we can show that, for $p \equiv 11$ $(\bmod 12), 47 \leq p \leq 197$, there are congruences of the form

$$
12 \theta \sum_{\alpha, \beta, \gamma} a_{\alpha, \beta, \gamma} P^{\alpha} Q^{\beta} R^{\gamma} \equiv Q^{p}\left(Q^{3}-R^{2}\right)^{p-b} \quad(\bmod p)
$$

which have the desired property. Indeed, $Q^{-p}$ is congruent modulo $p$ to a power series in $x^{p}$. So multiplying the right-hand side of (15) by $Q^{-p}$ preserves the divisibility by $p$ of the coefficients of $x^{n p}$. For example with $p=47, k=26, b=42$ we have

$$
\begin{gathered}
12 \theta \sum_{\alpha=0}^{11} \sum_{\substack{\beta=\alpha \\
\beta \equiv \alpha(\bmod 3)}}^{(123-\alpha) / 2} a_{\alpha, \beta} P^{\alpha} Q^{\beta} R^{(123-\alpha-2 \beta) / 3} \\
\equiv Q^{47}\left(Q^{3}-R^{2}\right)^{5} \quad(\bmod 47)
\end{gathered}
$$

where the coefficients $a_{\alpha, \beta}$ are given by Table 3 .

Of course the congruences in Table 1 are really statements about Cauchy powers of Ramanujan's $\tau$-function and can be established using modular function theory as already indicated. The author conjectures that, corresponding to every congruence of the form (1) there is a congruence $(13)$, except possibly when $p \equiv 11(\bmod 12)$ and $k=26$ in which case both (14) and (15) apply. 
TABLE 3

\begin{tabular}{|c|c|c|c|c|c|c|c|c|c|c|c|c|c|c|}
\hline$\beta$ & $\begin{array}{l}\alpha \\
0\end{array}$ & 3 & 6 & 9 & $\beta$ & $\begin{array}{l}\alpha \\
1\end{array}$ & 4 & 7 & 10 & $\beta$ & $\begin{array}{l}\alpha \\
2\end{array}$ & 5 & 8 & 11 \\
\hline 0 & 15 & & & & 1 & 6 & & & & 2 & 31 & & & \\
\hline 3 & 21 & 35 & & & 4 & 41 & 41 & & & 5 & 45 & 12 & & \\
\hline 6 & 7 & 32 & 3 & & 7 & 40 & 21 & 19 & & 8 & 41 & 42 & 20 & \\
\hline 9 & 21 & 0 & 8 & 33 & 10 & 38 & 2 & 27 & 4 & 11 & 33 & 16 & 20 & 30 \\
\hline 12 & 17 & 7 & 15 & 29 & 13 & 6 & 10 & 46 & 13 & 14 & 46 & 22 & 22 & 2 \\
\hline 15 & 19 & 23 & 13 & 31 & 16 & 11 & 0 & 40 & 2 & 17 & 22 & 36 & 9 & 2 \\
\hline 18 & 18 & 4 & 16 & 42 & 19 & 44 & 3 & 23 & 25 & 20 & 28 & 11 & 44 & 44 \\
\hline 21 & 45 & 15 & 29 & 9 & 22 & 23 & 31 & 45 & 29 & 23 & 37 & 14 & 31 & 28 \\
\hline 24 & 33 & 39 & 36 & 29 & 25 & 22 & 33 & 21 & 12 & 26 & 24 & 2 & 1 & 17 \\
\hline 27 & 41 & 1 & 35 & 17 & 28 & 37 & 8 & 25 & 25 & 29 & 5 & 28 & 41 & 43 \\
\hline 30 & 25 & 37 & 38 & 45 & 31 & 45 & 28 & 27 & 16 & 32 & 26 & 44 & 10 & 27 \\
\hline 33 & 26 & 15 & 3 & 27 & 34 & 18 & 38 & 4 & 46 & 35 & 9 & 39 & 25 & 8 \\
\hline 36 & 40 & 16 & 45 & 26 & 37 & 31 & 41 & 36 & 1 & 38 & 13 & 3 & 18 & 33 \\
\hline 39 & 33 & 1 & 34 & 14 & 40 & 12 & 33 & 3 & 3 & 41 & 38 & 27 & 28 & 46 \\
\hline 42 & 41 & 36 & 1 & 43 & 43 & 19 & 25 & 36 & 21 & 44 & 13 & 3 & 45 & 5 \\
\hline 45 & 32 & 34 & 14 & 44 & 46 & 2 & 31 & 25 & 5 & 47 & 33 & 39 & 35 & 43 \\
\hline 48 & 24 & 15 & 2 & 38 & 49 & 45 & 34 & 16 & 24 & 50 & 38 & 43 & 11 & 14 \\
\hline 51 & 4 & 0 & 45 & 22 & 52 & 22 & 46 & 18 & 29 & 53 & 14 & 16 & 28 & 44 \\
\hline 54 & 7 & 30 & 38 & 4 & 55 & 37 & 3 & 6 & 7 & 56 & 17 & 11 & 17 & 26 \\
\hline 57 & 30 & 29 & 7 & 23 & 58 & 25 & 6 & 42 & & 59 & 45 & 9 & & \\
\hline 60 & 29 & 16 & & & 61 & 13 & & & & & & & & \\
\hline
\end{tabular}

Further congruences can be established by the same method. For example each of the following functions is congruent modulo $p$ to a power series of the form $12 \theta f(x)$.

$$
\begin{array}{ll}
p=11: & \left(Q^{3}-R^{2}\right)^{8}+4 P^{6} Q^{18}\left(Q^{3}-R^{2}\right) \\
& \left(Q^{3}-R^{2}\right)^{10}+6 P^{8} Q^{23}\left(Q^{3}-R^{2}\right) \\
p=13: & \left(Q^{3}-R^{2}\right)^{9}+5 P^{2} Q^{5}\left(Q^{21}-R^{14}\right) \\
& Q^{13}\left(Q^{3}-R^{2}\right)^{7}+2 P^{4} Q^{11}\left(Q^{21}-R^{14}\right), \\
p=17: \quad & \left(Q^{3}-R^{2}\right)^{4}+3 P^{12} R^{2}\left(Q^{3}-R^{2}\right) \\
& \left(Q^{3}-R^{2}\right)^{5}+6 P^{7} Q^{7} R\left(Q^{3}-R^{2}\right) \\
& \left(Q^{3}-R^{2}\right)^{8}+10 P^{9} Q^{15} R\left(Q^{3}-R^{2}\right), \\
& \left(Q^{3}-R^{2}\right)^{11}+9 P^{11} Q^{23} R\left(Q^{3}-R^{2}\right) \\
& \left(Q^{3}-R^{2}\right)^{12}+10 P^{6} Q^{30}\left(Q^{3}-R^{2}\right) \\
& Q^{17}\left(Q^{3}-R^{2}\right)^{12}+12 P^{6} Q^{23}\left(Q^{27}-R^{18}\right), \\
p=19: \quad & \left(Q^{3}-R^{2}\right)^{13}+P^{2} Q^{5}\left(Q^{33}-R^{22}\right) \\
p=23: \quad & \left(Q^{3}-R^{2}\right)^{9}+4 P^{14} Q^{17}\left(Q^{3}-R^{2}\right), \\
& \left(Q^{3}-R^{2}\right)^{16}+21 P^{6} Q^{42}\left(Q^{3}-R^{2}\right), \\
& \left(Q^{3}-R^{2}\right)^{20}+4 P^{8} Q^{53}\left(Q^{3}-R^{2}\right)
\end{array}
$$




$$
\begin{aligned}
p=29: \quad & \left(Q^{3}-R^{2}\right)^{8}+19 P^{7} Q^{16} R\left(Q^{3}-R^{2}\right), \\
& \left(Q^{3}-R^{2}\right)^{13}+2 P^{9} Q^{30} R\left(Q^{3}-R^{2}\right), \\
& \left(Q^{3}-R^{2}\right)^{18}+20 P^{11} Q^{44} R\left(Q^{3}-R^{2}\right), \\
& \left(Q^{3}-R^{2}\right)^{20}+P^{6} Q^{54}\left(Q^{3}-R^{2}\right), \\
& \left(Q^{3}-R^{2}\right)^{25}+9 P^{8} Q^{68}\left(Q^{3}-R^{2}\right), \\
& Q^{29}\left(Q^{3}-R^{2}\right)^{6}+5 P^{12} Q^{38}\left(Q^{3}-R^{2}\right), \\
p=31: \quad & \left(Q^{3}-R^{2}\right)^{6}+29 P^{8} Q^{11}\left(Q^{3}-R^{2}\right), \\
& \left(Q^{3}-R^{2}\right)^{21}+2 P^{2} Q^{11}\left(Q^{51}-R^{34}\right), \\
& \left(Q^{3}-R^{2}\right)^{30}+16 P^{17} Q^{77} R\left(Q^{3}-R^{2}\right), \\
& \left(Q^{3}-R^{2}\right)^{4}+16 P^{9} Q^{3} R\left(Q^{3}-R^{2}\right), \\
& \left(Q^{3}-R^{2}\right)^{7}+32 P^{8} Q^{14}\left(Q^{3}-R^{2}\right), \\
& \left(Q^{3}-R^{2}\right)^{10}+36 P^{7} Q^{22} R\left(Q^{3}-R^{2}\right), \\
& \left(Q^{3}-R^{2}\right)^{25}+P^{2} Q^{17}\left(Q^{57}-R^{38}\right), \\
& \left(Q^{3}-R^{2}\right)^{11}+40 P^{7} Q^{25} R\left(Q^{3}-R^{2}\right), \\
& \left(Q^{3}-R^{2}\right)^{18}+30 P^{9} Q^{45} R\left(Q^{3}-R^{2}\right), \\
& \left(Q^{3}-R^{2}\right)^{25}+22 P^{11} Q^{65} R\left(Q^{3}-R^{2}\right), \\
& \left(Q^{3}-R^{2}\right)^{28}+4 P^{6} Q^{78}\left(Q^{3}-R^{2}\right), \\
\left.p=41{ }^{3}\right) & \left(Q^{3}-R^{2}\right)^{35}+34 P^{8} Q^{98}\left(Q^{3}-R^{2}\right), \\
& \left(Q^{3}-R^{2}\right)^{8}+8 P^{8} Q^{17}\left(Q^{3}-R^{2}\right), \\
& \left(Q^{3}-R^{2}\right)^{29}+4 P^{2} Q^{17}\left(Q^{69}-R^{46}\right), \\
& \left(Q^{3}-R^{2}\right)^{32}+34 P^{6} Q^{90}\left(Q^{3}-R^{2}\right), \\
& \left(Q^{3}-R^{2}\right)^{40}+25 P^{8} Q^{113}\left(Q^{3}-R^{2}\right) \\
p=43: 47 &
\end{aligned}
$$

and

$$
p=541:\left(Q^{3}-R^{2}\right)^{136} .
$$

Finally we have a general result:

Theorem. Suppose $p=6 t+1$ is prime. Then there exist integer coefficients $a_{\beta}$ such that

$$
\begin{aligned}
& 12 x \frac{d}{d x} \sum_{\substack{\beta, \gamma \\
2 \beta+3 \gamma=5 p}} a_{\beta} Q^{\beta} R^{\gamma} \\
& \quad \equiv\left(Q^{3}-R^{2}\right)^{(5 p+1) / 6}-\left(\begin{array}{c}
4 t \\
t
\end{array}\right) Q^{p-1}\left(Q^{3(p+1) / 2}-R^{p+1}\right) \quad(\bmod p) .
\end{aligned}
$$


Proof. If $\beta$ and $\gamma$ are related by $2 \beta+3 \gamma=5 p$ then

$$
12 x \frac{d}{d x} Q^{\beta} R^{\gamma} \equiv 4 \beta Q^{\beta-1} R^{\gamma-1}\left(Q^{3}-R^{2}\right) \quad(\bmod p) .
$$

Writing $w$ for the integer $\frac{5 p+1}{6}$, we have to solve the following set of congruences modulo $p$.

$$
\begin{aligned}
& 4 a_{1} \equiv(-1)^{w}, \\
& 16 a_{4}-4 a_{1} \equiv(-1)^{w-1}\left(\begin{array}{c}
w \\
w-1
\end{array}\right), \\
& 4(p-3) a_{p-3}-4(p-6) a_{p-6} \equiv(-1)^{3 t+2}\left(\begin{array}{c}
w \\
3 t+2
\end{array}\right), \\
&-4(p-3) a_{p-3} \equiv(-1)^{3 t+1}\left(\begin{array}{c}
w \\
3 t+1
\end{array}\right)+\left(\begin{array}{c}
4 t \\
t
\end{array}\right), \\
& 4(p+3) a_{p+3}-4\left(\begin{array}{c}
w \\
3 t
\end{array}\right), \\
& 4(p+6) a_{p+6}-4(p+3) a_{p+3} \equiv(-1)^{3 t-1}\left(\begin{array}{c}
w \\
3 t-1
\end{array}\right), \\
& 4 \frac{5 p-3}{2} a_{(5 p-3) / 2}-4 \frac{5 p-9}{2} a_{(5 p-9) / 2} \equiv-\left(\begin{array}{c}
w \\
1
\end{array}\right), \\
&-4 \frac{5 p-3}{2} a_{(5 p-3) / 2} \equiv 1-\left(\begin{array}{c}
4 t \\
t
\end{array}\right) .
\end{aligned}
$$

A solution is possible since

$$
\begin{aligned}
1-\left(\begin{array}{c}
w \\
1
\end{array}\right)+\cdots+(-1)^{3 t}\left(\begin{array}{c}
w \\
3 t
\end{array}\right)-\left(\begin{array}{c}
4 t \\
t
\end{array}\right) \\
\quad \equiv 1+\left(\begin{array}{l}
t \\
1
\end{array}\right)+\cdots+\left(\begin{array}{c}
4 t-1 \\
3 t
\end{array}\right)-\left(\begin{array}{c}
4 t \\
t
\end{array}\right)(\bmod p) \\
\quad=0 .
\end{aligned}
$$

\section{REFERENCES}

[1] A. O. L. Atkin, Proof of a conjecture of Ramanujan, Glasgow Math. J., 8 (1967), 14-32.

[2] A. O. L. Atkin and J. N. O'Brien, Some properties of $p(n)$ and $c(n)$ modulo powers of 13, Trans. Amer. Math. Soc., 126 (1967), 442-459.

[3] A. O. L. Atkin, Ramanujan congruences for $p_{-k}(n)$, Canad. J. Math., 20 (1968), 67-78. 
[4] F. G. Garvan, A simple proof of Watson's partition congruences for powers of 7 , J. Austral. Math. Soc. (Series A), 36 (1984), 316-334.

[5] B. Gordon, Ramanujan congruences for $p_{-k}\left(\bmod 11^{r}\right)$, Glasgow Math. J., 24 (1983), 107-123.

[6] M. D. Hirschhorn and D. C. Hunt, A simple proof of the Ramanujan conjecture for powers of 5, J. für Math., 326 (1981), 1-17.

[7] K. Hughes, (to appear).

[8] M. I. Knopp, Modular Functions in Analytic Number Theory, (Markham Publishing Company, Chicago, 1970).

[9] J. Lehner, Proof of Ramanujan's partition congruence for $11^{3}$, Proc. Amer. Math. Soc., 1 (1950), 172-181.

[10] M. Newman, Some theorems about $p_{r}(n)$, Canad. J. Math., 9 (1957), 68-70.

[11] _ Congruences for the coefficients of modular forms and some new congruences for the partition function, Canad. J. Math., 9 (1957), 549-552.

[12] S. Ramanujan, On certain arithmetical functions, Trans. Cambridge Philos. Soc., 22 (1916), 159-184.

[13] _ Some properties of $p(n)$, the number of partitions of $n$, Proc. Cambridge Philos. Soc., 19 (1919), 207-210.

[14] _ Congruence properties of partitions, Math. Z., 9 (1921), 147-153.

[15] _ Properties of $p(n)$ and $\tau(n) \ldots$, The Lost Notebook and Other Unpublished Papers (Narosa Publishing House, New Delhi, 1988).

[16] G. N. Watson, Ramanujans Vermutung über Zerfällungsanzahlen, J. für Math., 179 (1938), 97-128.

Received March 13, 1991 and in revised form January 17, 1992.

22 St. Albans Road

KINGSTON UPON THAMES

SURREY KT2 5HQ, ENGLAND 


\title{
PACIFIC JOURNAL OF MATHEMATICS
}

Founded by

\author{
E. F. BeCKeNBACH (1906-1982) F. Wolf (1904-1989)
}

\section{EDITORS}

\section{S. VARADARAJAN (Managing Editor) University of California Los Angeles, CA 90024-1555 vsv@math.ucla.edu \\ F. Michael Christ University of California Los Angeles, CA 90024-1555 christ@math.ucla.edu}

\section{Herbert Clemens}

University of Utah

Salt Lake City, UT 84112

clemens@math.utah.edu

\author{
THOMAS ENRIGHT \\ University of California, San Diego \\ La Jolla, CA 92093 \\ tenright@ucsd.edu \\ Nicholas ERcolani \\ University of Arizona \\ Tucson, AZ 85721 \\ ercolani@math.arizona.edu \\ R. FINN \\ Stanford University \\ Stanford, CA 94305 \\ finn@gauss.stanford.edu \\ VAUGHAN F. R. JONES \\ University of California \\ Berkeley, CA 94720 \\ vfr@math.berkeley.edu
}

\section{SUPPORTING INSTITUTIONS}

UNIVERSITY OF ARIZONA

UNIVERSITY OF BRITISH COLUMBIA

UNIVERSITY OF OREGON

CALIFORNIA INSTITUTE OF TECHNOLOGY

UNIVERSITY OF CALIFORNIA

UNIVERSITY OF MONTANA

UNIVERSITY OF NEVADA, RENO

NEW MEXICO STATE UNIVERSITY

OREGON STATE UNIVERSITY
SteVen KerckhofF

Stanford University

Stanford, CA 94305

spk@gauss.stanford.edu

MARTIN SCHARLEMANN

University of California

Santa Barbara, CA 93106

mgscharl@henri.ucsb.edu

HAROLD STARK

University of California, San Diego

La Jolla, CA 92093

\author{
STANFORD UNIVERSITY \\ UNIVERSITY OF HAWAII \\ UNIVERSITY OF UTAH \\ WASHINGTON STATE UNIVERSITY \\ UNIVERSITY OF WASHINGTON
}

UIV 


\section{PACIFIC JOURNAL OF MATHEMATICS}

Volume $158 \quad$ No. $1 \quad$ March 1993

Determinant identities

GEORGE W. Eyre ANDrEWS and WiLliam H. Burge

A spectral theory for solvable Lie algebras of operators

E. BOASSO and ANGEL RAFAEL LAROTONDA

Simple group actions on hyperbolic Riemann surfaces of least area

S. Allen Broughton

Duality for finite bipartite graphs (with an application to $\mathrm{II}_{1}$ factors)

MARIE CHODA

Szegő maps and highest weight representations

MARK GREGORY DAVIDSON and Ron STANKE

Optimal approximation class for multivariate Bernstein operators

ZEEV DitZIAN and XINLONG ZHOU

Witt rings under odd degree extensions

ROBERT FITZGERALD

Congruence properties of functions related to the partition function

ANTHONY D. FORBES

Bilinear operators on $L^{\infty}(G)$ of locally compact groups

Colin C. GRAham and Anthony To-Ming LAU

Nonuniqueness of the metric in Lorentzian manifolds

GEOFFREY K. MARTIN and GERARD THOMPSON

Index theory and Toeplitz algebras on one-parameter subgroups of Lie 189 groups

EFTON PARK 